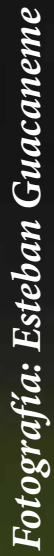

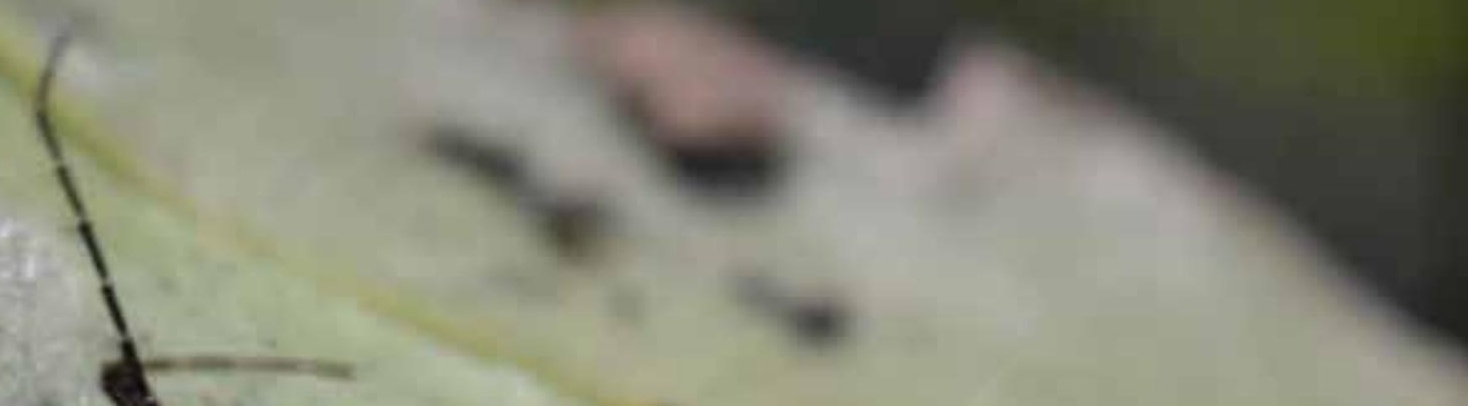

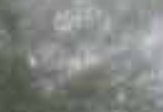
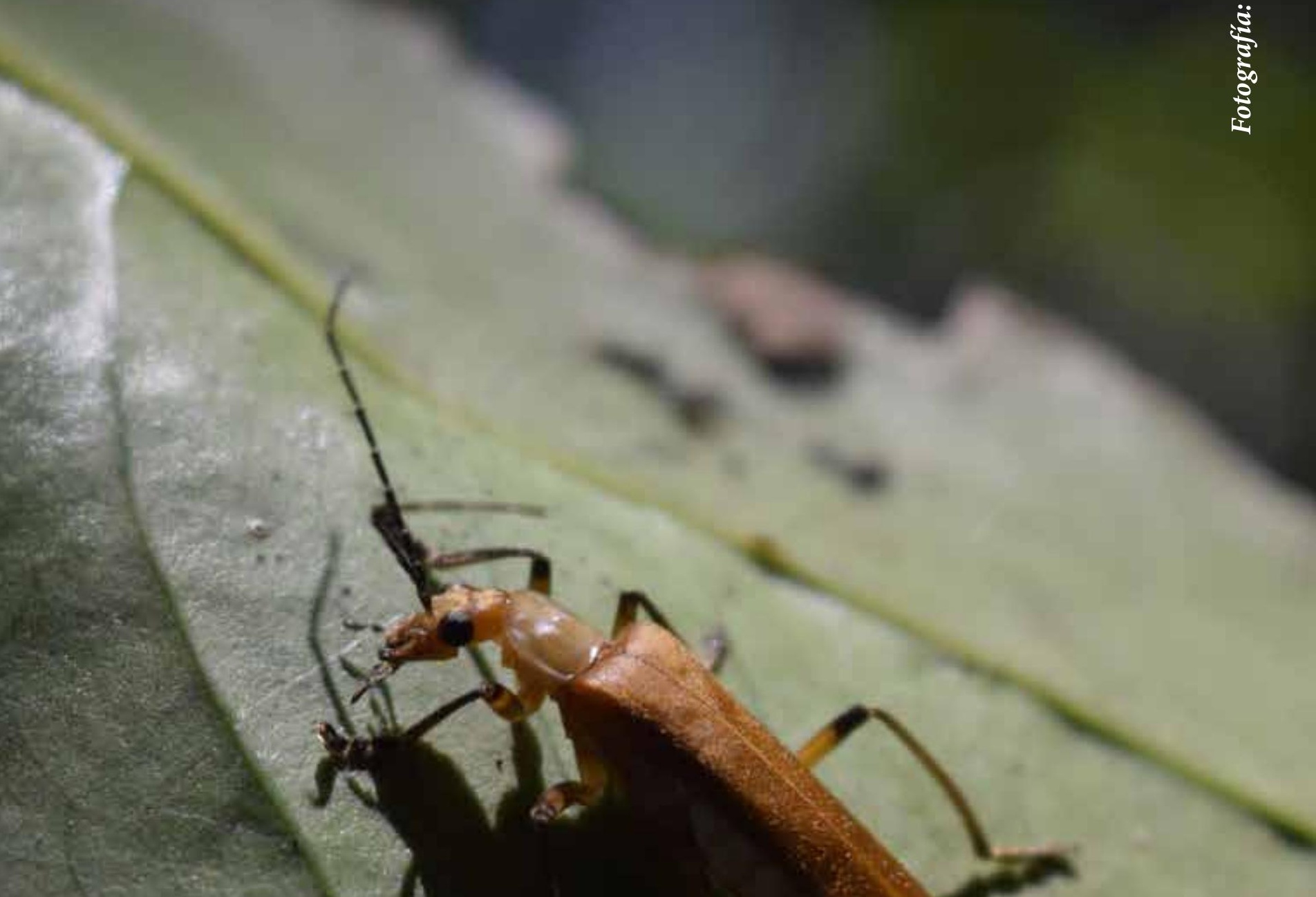


\title{
CORTOMETRAJES AMBIENTALES UTILIZADOS COMO HERRAMIENTAS PARA CONSTRUIR CULTURA AMBIENTAL EN ESTUDIANTES DE BACHILLERATO
}

\section{Environmental Short Films Used as Tools for Building Environmental Culture in High School Students}

\author{
Ángela Marcela Becerra Niño ${ }^{1}$ \\ Wilson Valderrama Hurtado ${ }^{2}$
}

Fecha de recepción: 5 de abril de 2016

Fecha de aprobación: 6 de octubre de 2016

\section{Resumen}

Este trabajo muestra una experiencia basada en la aplicación del proyecto de aula, en el cual se observó que los estudiantes del colegio Instituto Integrado de Comercio de Barbosa, Santander, tienen muy poca cultura sobre el cuidado del ambiente, pues se evidencia una mala actitud frente al respeto por la biodiversidad presente en la planta física de su institución. Por tal razón, se trabajó con herramientas pedagógicas como los cortometrajes ambientales para construir una cultura ambiental, pretendiendo que los estudiantes se concienciaran sobre los problemas que afectan día a día el bienestar de la madre tierra y lleven a cabo pequeñas acciones como el ahorro de agua, botar la basura en su lugar, ayudar a cuidar y valorar la fauna y flora presente en su colegio y, sobre todo, que transmitan dichas acciones a sus compañeros y sus familiares. Esta herramienta fue de gran utilidad, pues no se empleó mucho tiempo e hizo que los estudiantes, de una forma divertida, reconocieran muchos de los problemas ambientales que surgen hoy día, como la deforestación, la contaminación, el calentamiento global, etc., y eso permitió que al finalizar las presentaciones de los cortos, entablaran discusiones sobre sus acciones, para que puedan transformar positivamente sus estilos de vida y aportar de esa manera a un ambiente sano para las generaciones venideras.

Palabras clave: biodiversidad, herramientas pedagógicas, cultura ambiental, problemas ambientales

\section{Abstract}

This work shows an experience based on the application of the classroom project, in which it was observed that the students of the Institute Integrated School of Commerce of Barbosa, Santander, have very little culture for the care of the environment, since they have a bad attitude towards the respect for the biodiversity present in the physical plant of his institution. For this reason, we worked with pedagogical tools such as environmental shorts to build an environmental culture, pretending that students become aware of the problems that affect the well-being of mother earth day and carry out small actions such as saving water, throwing garbage in their place, help to care for and value the fauna and flora present in their school and, above all, to transmit these actions to their colleagues and their families. This tool was very useful because it did not take much time and made students, in a fun way, recognize many of the

1 Licenciada en Ciencias Naturales y Educación Ambiental, Universidad Pedagógica y Tecnológica de Colombia.

2 Licenciado en Ciencias Naturales y Educación Ambiental, Universidad Pedagógica y Tecnológica de Colombia. 
environmental problems that arise today, such as deforestation, pollution, global warming, etc., and This allowed them to finish the presentations of the shorts, to start discussions about their actions, so that they can positively transform their lifestyles and thus contribute to a healthy environment for future generations.

Keywords: biodiversity, educational tools, environmental awareness, environmental problems

\section{Introducción}

La utilización de herramientas como los cortometrajes ambientales permite que los estudiantes de grados séptimo, octavo y noveno del colegio Instituto Integrado de Comercio, de Barbosa (Santander), se culturicen ambientalmente, pues hoy día nuestro planeta enfrenta una crisis que afecta tanto a ricos como a pobres, debido al proceso de transformación de la naturaleza emprendido por el ser humano con el objetivo de extraer recursos y, principalmente, a la indiferencia por el daño que hacemos día a día al ambiente. Ello trae como consecuencias la contaminación del aire, los mares y los ríos, la pérdida de hábitats naturales y de especies, y un largo etcétera, que influyen negativamente sobre nosotros, nuestra salud y nuestra calidad de vida, y también sobre las generaciones venideras (Bordehore, 2006).

Por lo anterior, se necesita culturalizar hacia el cuidado del ambiente; sobre todo, a los niños y los jóvenes, pues el futuro de la tierra está en manos de ellos; por tal razón, es indispensable que los profesores de ciencias naturales y educación ambiental trabajen en la necesidad de promover entre los jóvenes acciones que conlleven un pensamiento crítico de conservación de la fauna, la flora, los ecosistemas y todos los componentes de la biodiversidad.

Es importante buscar estrategias que permitan acercar a los estudiantes a la responsabilidad por el cuidado del ambiente, y que dichas estrategias sean llamativas y motivadoras, y, sobre todo, que tengan un impacto favorable en la culturalización por el respeto a la naturaleza. Por ende, este trabajo tuvo como finalidad la utilización de cortometrajes ambientales como herramientas pedagógicas que permitan incentivar la cultura ambiental en estudiantes de bachillerato pertenecientes al grado noveno del colegio Instituto Integrado de Comercio, de Barbosa, Santander, para hacerlos más conscientes de las actitudes negativas como el excesivo consumismo que ejercen diariamente en sus vidas cotidianas.

\section{Metodología}

Este trabajo es de tipo descriptivo, pues busca especificar propiedades importantes de un conjunto de individuos, de una población o de un área de interés y se vale de técnicas estadísticas descriptivas para observar, organizar, concentrar, visualizar, comparar y presentar los datos (Ramírez, Arcila, Buritacá y Castrillón, 2004; Sampieri, Fernández y Baptista, 2007). En este caso, mide y evalúa las respuestas de los estudiantes en el debate que se hace al finalizar la presentación de cada uno de los cortometrajes ambientales, presentados los lunes durante cuatro meses con 35 estudiantes de grado noveno; así pues, esta sería una investigación descriptiva de conjunto sobre actitudes y opiniones de una muestra representativa de la población (Achaerandio, 2010).

\section{Institución participante}

El colegio participante es el Instituto Integrado de Comercio, sede A; está ubicado en la vereda Cristales, $\mathrm{Km} 1$ vía Barbosa-Puente Nacional, y alberga a estudiantes de los grados sexto a once.

En la figura 1 se observa parte de las instalaciones del colegio.

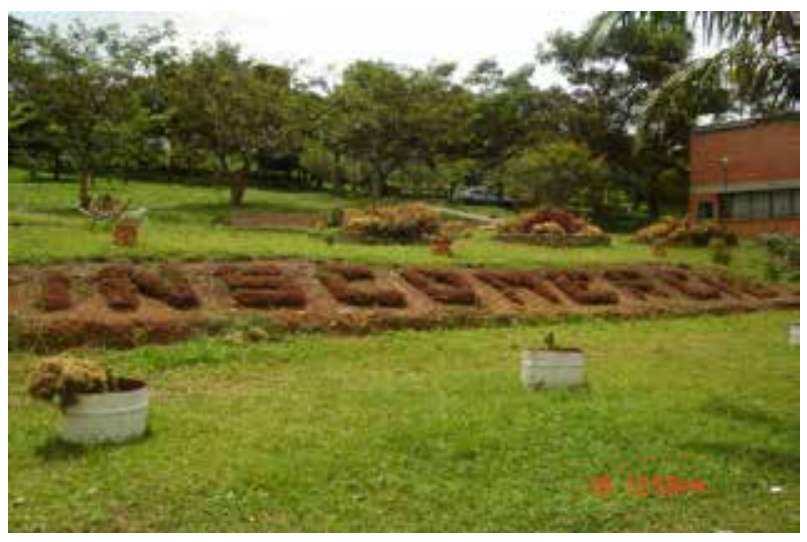

Figura 1. Sede A, Colegio Instituto Integrado de Comercio. Fuente: fotografía recuperada de: http://inscomerciosedea.blogspot. com/2011/05/como-describir-nuestro-colegio.html

\section{Fase 1. Presentación de cortometrajes ambientales}

En la tabla 1 se muestra el cuestionario que se respondía antes de ver los cortometrajes. Una vez resuelto el cuestionario guía, se dio inicio a la presentación y la explicación de los cortometrajes. 
Tabla 1. Cuestionario guía, proyecto de aula

\begin{tabular}{|l|l|}
\hline \multicolumn{2}{|c|}{ Introducción al proyecto de aula Cortitos Ambientales } \\
\hline Pregunta & \\
\hline ¿Qué es ambiente? & \\
\hline ¿Cuáles problemas ambientales enfrentamos hoy día? Nombre al menos dos. & \\
\hline ¿Cómo podemos solucionar los problemas ambientales? & \\
\hline ¿Qué es un cortometraje? & \\
\hline ¿Qué es un cortometraje ambiental? & \\
\hline
\end{tabular}

Fuente: Elaboración propia

\section{Fase 2. Visualización de cortometrajes}

Cada semana se les presentaban a los estudiantes uno o dos cortometrajes, faltando 15 minutos para terminar la clase.

\section{Fase 3. Discusión sobre la temática del cortometraje}

Luego de la presentación, los estudiantes comentaban sobre la temática aportando opiniones o sugerencias y dando respuesta a las preguntas establecidas.

En la tabla 2 se dan a conocer los cortometrajes ambientales que se visualizaron y las preguntas que se fomentaron al final de cada presentación.

Tabla 2. Cortometrajes ambientales presentados

\begin{tabular}{|l|l|l|}
\hline \multicolumn{1}{|c|}{ Fecha } & \multicolumn{1}{|c|}{ Video } & \multicolumn{1}{c|}{ Preguntas } \\
\hline 06 de abril & Tierra el llamado. Duración: 11:01 min. & ¿Qué mensaje nos muestra el video? ¿Qué piensas al respecto? \\
\hline 13 de abril & Choiba. Duración: 5:22 min. & $\begin{array}{l}\text { ¿Qué pasa con las especies que habitan el bosque? } \\
\text { ¿Será que, en el video, solo el mono araña está en riesgo de extin- } \\
\text { ción? }\end{array}$ \\
\hline 20 de abril & Día de La Tierra 2014. Duración 2:50 min & ¿Qué nos muestra el video? \\
\hline 11 de mayo & $\begin{array}{l}\text { Contaminación del mundo animado. Duración: 3:35 min.; } \\
\text { y Los animales salvan el planeta. Duración: 7:36 min. }\end{array}$ & $\begin{array}{l}\text { ¿Qué nos quiere mostrar el video } 1 ? \\
\text { ¿Será que, como seres "superiores" a los animales podemos tomar } \\
\text { algunas de sus acciones para cuidar el planeta? }\end{array}$ \\
\hline 18 de mayo & $\begin{array}{l}\text { Creando conciencia. Duración: 4:04 min. } \\
\text { ¿Cuál es el principal problema que se presenta en el video? } \\
\text { ¿Será que nosotros podemos cambiar nuestra actitud frente a este } \\
\text { problema? }\end{array}$ \\
\hline 01 de junio & $\begin{array}{l}\text { Salvemos al mundo. Duración: 4:13 min.; y Second Wind. } \\
\text { Duración: 5:50 min. }\end{array}$ & $\begin{array}{l}\text { Despertar. Duración: 2:00 min.; y Off. Duración: 2:55 min. } \\
\text { dejaremos a nuestras futuras generaciones? ¿De hoy en adelante } \\
\text { serás más responsable con el cuidado del planeta? }\end{array}$ \\
\hline
\end{tabular}

Fuente: Elaboración propia 


\section{Fase 4. Ideas para contribuir con el cuidado del ambiente}

Luego de cada discusión, surgían ideas para realizar campañas en pro del cuidado del ambiente, tales como "apaga las luces que no necesites", "no malgastes el agua", "sembremos un árbol" o "usemos bolsas de tela para hacer mercado".

\section{Fase 5. Pregunta de finalización}

Se preguntó a los estudiantes: ¿Los motivó la presentación de cortometrajes ambientales, para cuidar y valorar los recursos naturales? Esto, con el fin de describir las percepciones de ellos frente al uso de cortometrajes.

\section{Resultados}

La presentación de los cortometrajes permitió, de una forma divertida, llamativa y que no requería mucho tiempo para su presentación, que los estudiantes apren- dieran sobre los problemas ambientales que los afectan hoy día, como el calentamiento global, la extinción de especies, etc., y que ejerzan acciones pequeñas, pero significativas, como un cambio de actitud positiva y responsable frente al cuidado del ambiente: por ejemplo, la utilización de bolsas de tela para el mercado, el ahorro de energía, el ahorro de agua, la siembra de árboles y la lucha por la no explotación minera en los páramos, entre otros. Todo esto permitió que algunos de ellos tomaran conciencia y tuvieran una percepción distinta sobre la importancia de la naturaleza, y se conectaran con ella; según Bayón y Morejón (2006), el desarrollo de una cultura ambiental supone un cambio de concepción del hombre sobre sí mismo y sobre su lugar en el mundo, y, consecuentemente, sobre su lugar respecto a los otros seres humanos, la sociedad y la naturaleza.

Frente a la pregunta cerrada: ¿Los motivó la presentación de cortometrajes ambientales para cuidar y valorar los recursos naturales?, obtuvimos la siguiente gráfica (figura 2):

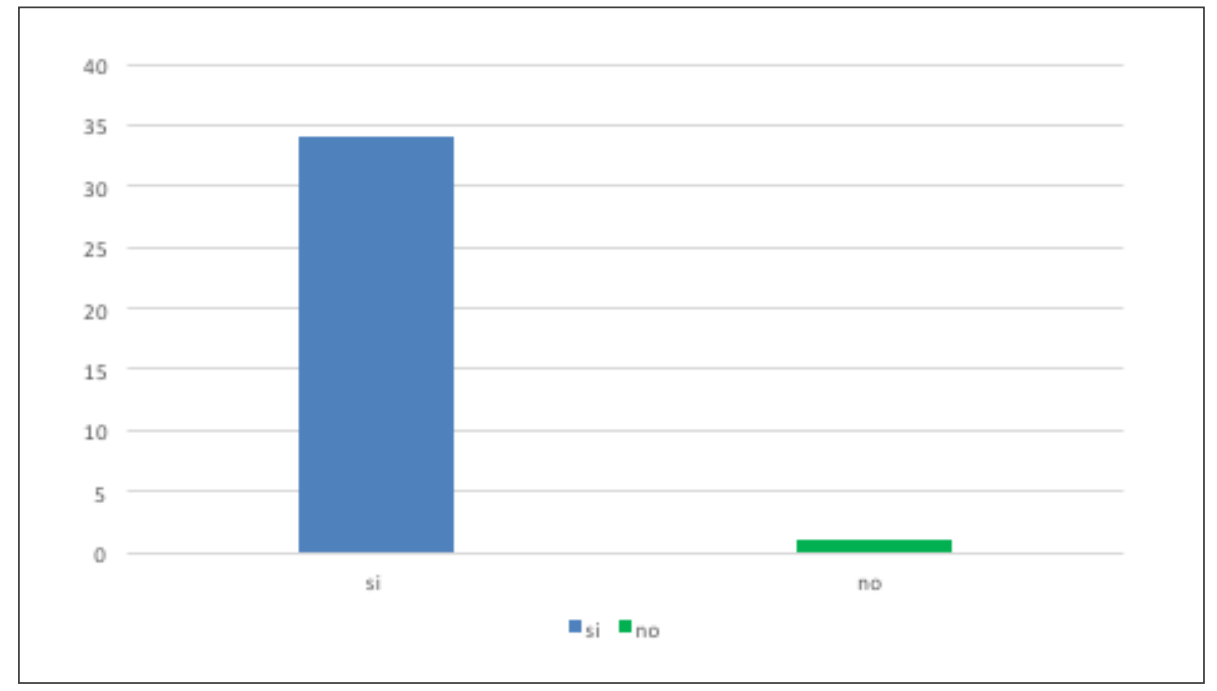

Figura 2. Porcentajes de respuestas por parte de los estudiantes a la pregunta: ¿Los motivó la presentación de cortometrajes ambientales para cuidar y valorar los recursos naturales?

La figura 2 muestra que de 35 estudiantes, 34 respondieron afirmativamente; por ende, haber utilizado los cortometrajes ambientales motivó a los estudiantes para cuidar, indagar y dar a conocer los problemas ambientales que nos aquejan hoy día; por tal razón, es pertinente seguir creando estrategias que permitan el conocimiento sobre estos problemas, para poder encontrarles una posible solución; en este caso, culturalizando ambientalmente no solo a los estudiantes, sino, por medio de ellos, también a la gente del común, como los padres de familia, así sea un porcentaje mínimo de ellos.
Finalmente, algunas de las opiniones de los estudiantes tras la presentación de algunos de los cortometrajes fueron: "Lo que más me llamó la atención fue ver cómo el planeta se está acabando, llegando a su extinción la naturaleza; y todo esto se ve muy mal y sucede por culpa del hombre" (estudiante Natalia Casas). "Los cortometrajes me gustaron, porque nos muestra una realidad que va a suceder, cómo en algunos años nosotros nos vamos a quedar sin el oxígeno que los árboles nos dan, por culpa de la humanidad. En el video se ve que el hombre respiraba por una máquina de oxígeno, y que lloraba al recordar 
cómo era respirar debajo de los árboles" (estudiante Diego Zapata).

\section{Conclusiones}

Es importante llevar a cabo proyectos para concienciar a los estudiantes sobre el cuidado del ambiente, y hacerlo desde edades tempranas, puesto que en los estudiantes de bachillerato la percepción de los problemas ambientales no es muy alentadora, y casi no tiene relevancia en su vida cotidiana.

El uso de cortometrajes ambientales como herramienta de enseñanza permitió que los educandos conozcan, discutan y reflexionen sobre los problemas que aquejan la vida de la naturaleza, y, por ende, la de los seres humanos, utilizando poco tiempo y de forma que hacerlo conlleve ejercer acciones de cambio frente a las actitudes negativas en contra del cuidado del ambiente.

El fomento de los medios audiovisuales para explicar temas como el calentamiento global, la pérdida de la diversidad natural, la deforestación, la contaminación, etc., ayuda a que los estudiantes tengan una mejor percepción de lo que sucede con el ambiente, debido a las escenas llamativas que muestran los cortometrajes.

\section{Referencias}

Achaerandio, L. (2010). Iniciación a la práctica de la investigación. Universidad Rafael Landivar 7 edición actualizada, 23-28.

Bayón, P. y Morejón, A. (2006). Cultura ambiental y la construcción de entornos de reproducción social en Cuba: un reto para el siglo XXI. Calzada, 251.

Bordehore, C. (2006). Problemas ambientales, problemas humanos. Recuperado de http://www. citethisforme.com/es/cite/ebook

Ramírez, L., Arcila, A., Buritacá, L. y Castrillón, J. (2004). Paradigmas y modelos de investigación: guía didáctica y módulo. Recuperado de http://virtual.funlam.edu.co/repositorio/sites/default/files/ repositorioarchivos/2011/02/0008paradigmasymodelos.771.pdf

Sampieri, R., Fernández, C. y Baptista, P. (2007). Fundamentos de metodología de la investigación. Madrid: McGraw-Hill.

\section{Cibergrafía}

http://inscomerciosedea.blogspot.com/2011/05/como-describir-nuestro-colegio.html 